\title{
Study of Black Cotton Soil Characteristics with Molasses
}

\author{
S. Vinodhkumar ${ }^{1}$, P. Kulanthaivel ${ }^{2}$, A. Kabilan ${ }^{3}$ and K. M. Lokeshkanna ${ }^{4}$ \\ ${ }^{1 \& 2}$ Assistant Professor, ${ }^{3 \& 4}$ UG Scholar, Kongu Engineering College, Perundurai, Tamil Nadu, India \\ E-Mail: shansvino@gmail.com, kulanthaivel.civil@kongu.ac.in, kabilan.kabil5@gmail.com, \\ lokeshkannakm@gmail.com
}

\begin{abstract}
Civil engineering structure resting on expansive soil needs more attention since it causes undesirable engineering behavior when the soil comes in contact with water. The areas consisting of expansive soil need a proper stabilization method to solve the site problems faced by the civil engineers. Pavements constructed on such soil shows signs of damage continuously during the service life of the pavement eventually increase the maintenance costs. Stabilization of this soil is a usual practice for improving the strength. In this work an attempt was made on the utilization of Molasses with soil by adding various percentages of 5 , $7.5,10,12.5$ and $15 \%$ in soil and accessed their geotechnical behavior for different curing periods. The effects of Molasses treatment on the strength improvement of compacted expansive clays are studied based on Unconfined Compression tests for different curing periods of 0,7 and 14 days. UCS performed on these mixes and identified that addition of Molasses attained high strength with the increasing percent of Molasses in soil. From the test results, it was observed that the UCS peak value were increases to $160 \%$. From the experimental study, it is observed that the treatment of the expansive soil with the addition of Industrial waste material Molasses is more efficient in subgrade modification and other geotechnical applications.
\end{abstract}

Keywords: Molasses, Stabilization, UCS

\section{INTRODUCTION}

This project is done because due to increasing urbanization, land for construction is getting scarcer and now is very important to utilize the limited land. For centuries mankind was wondering at the instability of earth materials, especially expansive soil. One day they are dry and hard, and the next day wet and soft. Swelling soil always create problem for lightly loaded structure, by consolidating under load and by changing volumetrically along with seasonal moisture variation. As a result the pavements usually counter excessive settlement and differential movements, resulting in damage to foundation systems, structural elements and architectural features. In a significant number of cases the structure becomes unstable or uninhabitable. Even when efforts are made to improve swelling soil, the lack of appropriate technology sometimes results volumetric change that are responsible for billion dollars damage each year. It is due to this that the present work is taken up. The purpose was to check the scope of improving bearing capacity value and reduce expansiveness by adding additives. There are number of additives for soil modification like ordinary Portland cement, fly ash, lime fly ash etc. Molasses is a waste product obtained from sugar industries are being effectively used here as a cost effective and eco-friendly stabilizing material. As the molasses is poured over the agricultural land it improves strength of the soil and increases agricultural productivity.

\section{LITERATURE REVIEW}

Vinodhkumar S [1] (2015), explains the effectiveness of uses of flyash as soil stabilizer i.e. flyash for improving the shear strength and CBR value of two types of fine grained soils. In case of flyash, the experimental variables were soil type (intermediate compressible clay and highly compressible clay), amount of flyash and treatment duration. The results showed that with use liquid stabilizer, there was appreciable increment in unconfined compressive strength and CBR value of both soils. The unconfined compressive strength of soil increased with increment ratio in range of 1.57-2.01 for both types of soils. CBR value of soils had increment ratio of $2-3.5$ in both types of soils. The values increased with increase in treatment duration. Based on this study, optimum amount of liquid additive to be added to respective soil types for higher strength increments were determined.

Neva Elias [2] (2015), investigated the use of waste materials in geotechnical application sand to evaluate the effects of waste paper sludge on strength development of soft soil. This review discusses the effect of waste paper sludge on stabilized soils. In this paper, attempts are made to utilize the same for the soil improvement. The application of Waste Paper Sludge (WPS) will be investigated in this study by conducting laboratory tests, compaction and unconfined compressive strength. Soil with $2 \%$ and 5\% WPS have an optimum moisture content more closed to OMC of clay soil alone. The addition of WPS has increased the strength at $5 \%$ and it was found to be a constant and optimum value of strength to soil. In general it was found that WPS is a suitable waste material for strengthening soft soil. The beneficial reuse of the paper sludge also saved landfill space.

M. A`dams Joe \& A. Maria Rajesh [3] (2015), have investigated that stabilization of soil using industrial waste. Unsuitable highway sub grade soil requires stabilization to 
improve its properties. Industrial waste sand is used as raw materials when the sand can no longer be reused in the industry, it is removed from the industry and is removed from the industry and is termed as industrial waste sand. Ingredients used are Copper slag, cement and lime. Copper slag is a by-product of Copper industry Lime was bought from locally available chemical laboratories. The project are planned to conduct various experiment like Specific gravity, sieve analysis, proctor compaction test, unconfined compressive strength and CBR test to increase strength properties and behavior of sub base. Then the results and graphs of various mixes are compared to see their effects in sub base stabilization. The stabilization technique has an additional benefit of providing an environment friendly way to deal with industrial waste sand.

Zamri bin chik et al., [4] (2007), have investigate the effect of adding different chloride compounds including $(\mathrm{NaCl}$, $\mathrm{MgCl} 2, \mathrm{CaCl} 2)$ on the engineering properties of silty clay soil. Various amounts of salts $(2 \%, 4 \%$, and $8 \%)$ were added to the soil to study the effect of salts on the compaction characteristics, consistency limits and compressive strength. The main findings of this study were that the increase in the percentage of each of the chloride compounds increased the maximum dry density and decrease the optimum moisture content. The liquid limit, plastic limit and plasticity index decreased with the increase in salt content. The unconfined compressive strength increased as the salt content increased.

Vinodhkumar S [5] (2015), have explains about infrastructure project for instance highways, railways, water reservoirs, reclamation etc., requires earth material in very huge quantity. In urban areas, borrow earth is not easily available which has to be hauled from an elongated distance. Fairly often large areas are covered with highly plastic and clayey soil, which is not suitable for such purpose. Extensive laboratory and field trials have been carried out by various researchers and have shown promising results for application of such expansive soil after stabilization with traditional stabilizing agents such as cement, lime, bitumen etc. The growing cost of traditional stabilizing agents and the need for the economical utilization of industrial and agricultural wastes has prompted an investigation into the stabilizing prospective of coal ash, groundnut shell ash and bagasse ash in highly expansive soil. In this study, an attempt has been made to utilize the industrial and agricultural wastes such as coal ash, groundnut shell ash and bagasse ash as stabilizing agent. The effect of industrial and agricultural wastes under equal proportioning on certain properties of soil such as Optimum Moisture Content, Maximum Dry Density, Unconfined Compressive Strength (UCC) and California Bearing Ratio (CBR) has been studied under both light and heavy compaction. It has been observed that 12\%C.A + $12 \%$ GSA, 16\%C.A + 16\%B.A and 16\%B.A + 16\%GSA are the optimum percentage combinations, which give the maximum CBR value. The CBR value increased from $1.63 \%$ for native soil to $7.87 \%, 6.79 \%$ and $7.88 \%$ for respective combinations. The proportion increase was found to be $382.82 \%, 316.56 \%$ and $383.44 \%$.

K. Mahendran et al., [6] (2016) have made an attempt to investigate the use of hypo sludge with cement and molasses for the preparation of stabilized red mud blocks. The Laboratory test results show that the physical and the mechanical properties of the red soil were assessed. The unconfined compressive strength, modulus of rupture, Ultrasonic Pulse Velocity (UPV) and water absorption tests were performed for this soil with and without hypo sludge, cement and molasses. It has been identified that $15 \%$ of Hypo Sludge with Cement and Molasses as additives provides optimum stabilization to the soil and it is proven with the help of unconfined compression test.

Monica Malhotra [7] (2013), have described about the foundation is very important and it has to be strong to support the entire structure. In order for the foundation to be strong, the soil around it plays a very critical role. To work on soils, we need to have proper knowledge about their properties and factors which affect their behavior. Expansive soils always create problems more for lightly loaded structures than moderately loaded structures. By consolidating under load and changing volumetrically along with seasonal moisture variation, these problems are manifested through swelling, shrinkage and unequal settlement. In this paper the experimental results obtained in the laboratory on expansive soils treated with low cost materials (lime and flyash) are presented. A study is carried out to check the improvements in the properties of expansive soil with fly ash and lime in varying percentages. The test results such as liquid limit, standard proctor compaction, and differential free swelling test obtained on expansive clays mixed at different proportions of lime and fly ash admixture are presented and discussed in this paper. The results show that the stabilized clay has lesser swelling potential whereas increase in optimum moisture content has been observed.

Ajesh K. Kottuppillil et al., [8] (2016) have explained about the civil engineering structure resting on expansive soils needs more attention since it causes undesirable engineering behavior when the soil comes in contact with water. The areas consisting of expansive soil need a proper stabilization method to solve the site problems faced by the civil engineers. Pavements constructed on such soil shows signs of damage continuously during the service life of the pavement eventually increase the maintenance costs. Stabilization of these soils is a usual practice for improving the strength. In this work an attempt was made on the utilization of Phospho Gypsum (PG) with soil by adding various percentages of 2,4 , and $6 \%$ in soils and accessed their geotechnical behavior for different curing periods. The effects of PG treatment on the microstructure and strength improvement of compacted expansive clays are studied based on Microstuctural analysis and Unconfined Compression tests for different curing periods of 0,14 and28 days. UCS performed on these mixes and identified 
that addition of phosphogypsum attained high strength with the increasing percent of phosphogypsum in soils. From the test results, it was observed that the UCS peak stresses were increases to $162 \mathrm{kPa}$ and $336 \mathrm{kPa}$ from $75 \mathrm{kPa}$ and $122 \mathrm{kPa}$ for soil samples S1 and S2 at 28days of curing. X-ray Diffraction and Scanning Electron Microscopy were carried out for micro level analysis and the results confirmed the presence of minerals and cementation nature in the treated soil. From the experimental study, it is observed that the treatment of the expansive soil with the addition of Industrial waste material Phosphogypsum is more efficient in sub grade modification and other geotechnical applications.

Debangana Sharmah [9] (2016), have suggested that the expansive soils are very hard when dry, but loses its strength completely in wet condition. These expansive soils pose several problems for the civil engineers. Various methods are adopted to improve the engineering characteristics of expansive soils. Soil stabilization is one of the important features for construction because it improves the engineering properties of soil such as strength, durability and volume stability. In this paper, sugarcane straw ash is used at varying percentage and at varying curing periods to stabilize the soil. Various geotechnical laboratory tests like Unconfined Compression Test (UCS), California Bearing Test (CBR) and Free Swelling Index Test (FSI) were carried by varying the percentage of sugarcane straw ash (5\%, 10\% and $15 \%)$ at varying curing periods (3, 5and 7 days).

It is found that $10 \%$ increase in the percentage of sugarcane straw ash increases the UCS and CBR value with increasing curing periods.10. Dallas and Syam (2009) have studied the process of stabilization with the traditional calcium-based stabilizers, Portland cement, lime, and Fly ash. The report describes and compares the basic reactions that occur between these stabilizers and soil and the mechanisms that result in stabilization. The report presents a protocol a protocol for each stabilizer through which the selection of the stabilizer is validated based on the tests conducted on mixes. The mixture design process defines an acceptable amount of stabilizer for the soil in question based on consistency testing, strength testing, and in some cases (resilient) modulus testing. Within each additive validation and mixture design protocol, an assessment of the deleterious soil additive reactions was made.

Ramya [10] (2017), have described application of support vector machines based on bound theorems. The application of SVMs in stability analysis is studied and presented in this paper. The use of SVMs is very advantageous for the prediction of FoS because it can perform nonlinear regression efficiently for high dimensional data sets. As the SVM is mainly branded by the type of its kernel function, it is indispensable to elect the proper kernel for each precise application problem in order to promise acceptable results. The outcome of sigmoid function shows that SVMs have the capability to calculate the FoS with an adequate degree of exactness.

G. V. Rama Subbarao [11], (2011), have described the Soil existing at a particular site may not be appropriate for construction of engineering structures. The present study made an attempt to enhance the geotechnical properties of a soil replaced with industrial wastes having pozzolanic value like rice husk ash (RHA) and fly ash (FA). Soil is replaced with RHA in $2 \%, 4 \%$, and $6 \%$ to dry weight of soil. It is observed that soil replaced with $4 \%$ RHA is the optimum for the soil used in this study from geotechnical point of view. To know the influence of fly ash, soil is further replaced with 4\% FA along with 4\% RHA. It is found that results of soil replacement by both RHA and FA proved to be soil modification and not the improvement. Hence, a cost-effective accelerator like lime is used for further replacing the above soil-4\%, RHA-4\% FA mix. The optimum lime content is found to be $4 \%$.

\section{EXPERIMENTAL INVESTIGATION}

\section{A. Materials}

The soil samples were excavated from the ground level after removing the top soil. The Collected soils were air dried, then pulverized and used for testing. An admixture is also collected and tested. The various materials were listed below are as follows

\section{B. Expansive soil}

Expansive soil are collected based on Free swell index. For obtaining expansive soil, 5 samples are collected from Coimbatore and free swell index were done on those samples as per IS 2720 (part 40) and finally expansive soil (of medium expansiveness) are collected from one place called kadaiyampatti.

\section{Molasses}

Molasses is a very thick, dark brown, syrupy liquid obtained as a by-product in processing cane sugar. It is also called treacle. It contains resinous and some inorganic constituents that render it unfit for human consumption. The molasses are collected from the sugar industry near erode.

\section{METHODOLOGY}

The project deals with stabilization of expansive soil by using molasses, Initially soil samples were collected from various places and it is categorized into treated and untreated soil . For untreated soil basic test were carried out like index properties , free swell index etc., and for treated soil molasses (admixture) were added and test were conducted based on the various percentage of molasses at 0 , 7 and 14 days curing period. 
TABLE I PROPERTIES OF SOIL

\begin{tabular}{|c|c|}
\hline Properties of Soil & Values \\
\hline Specific gravity & 2.7 \\
\hline Liquid Limit & $51 \%$ \\
\hline Plastic limit & $44 \%$ \\
\hline Optimum moisture content & $24 \%$ \\
\hline Maximum dry density & $1.74 \mathrm{~g} / \mathrm{cc}$ \\
\hline Unconfined compressive test & $47 \mathrm{KPa}$ \\
\hline
\end{tabular}

TABLE II SUMMARY OF RESULT

\begin{tabular}{|c|c|c|c|c|c|}
\hline \multirow{2}{*}{ Curring Period } & \multicolumn{7}{|c|}{ \% of Molasses } \\
\cline { 2 - 6 } & $5 \%$ & $7.5 \%$ & $10 \%$ & $12.5 \%$ & $15 \%$ \\
\hline \multicolumn{7}{|c|}{ Free Swell Index Test } \\
\hline 0th Day & 60 & 55 & 44 & 42 & 63 \\
\hline 7th Day & 45 & 40 & 37 & 35 & 56 \\
\hline 14thDay & 38 & 30 & 26 & 23 & 50 \\
\hline \multicolumn{7}{|c|}{ UCS Test } \\
\hline 0th Day & 0.045 & 0.072 & 0.088 & 0.110 & 0.055 \\
\hline 7th Day & 0.048 & 0.074 & 0.103 & 0.118 & 0.070 \\
\hline 14thDay & 0.074 & 0.089 & 0.109 & 0.122 & 0.081 \\
\hline \multicolumn{7}{|c|}{ Atterberg's Plastic Limit } \\
\hline 0th Day & 20 & 23 & 28 & 29 & 26 \\
\hline 7th Day & 22 & 26 & 30 & 28 & 28 \\
\hline 14thDay & 24 & 28 & 31 & 30 & 32 \\
\hline \multicolumn{7}{|c|}{ Atterberg's Liquid Limit } \\
\hline 0th Day & 45 & 42 & 38 & 40 & 40 \\
\hline 7th Day & 42 & 40 & 35 & 34 & 37 \\
\hline 14thDay & 40 & 37 & 33 & 32 & 34 \\
\hline
\end{tabular}

\section{A. Variation of Atterberg's limits in Free Swell Index Test}

It can be observed from table that the Atterberg's limit decreases from initial percentage to $12.5 \%$. And there is sudden increase in Atterberg's limit at reaching 15\%.

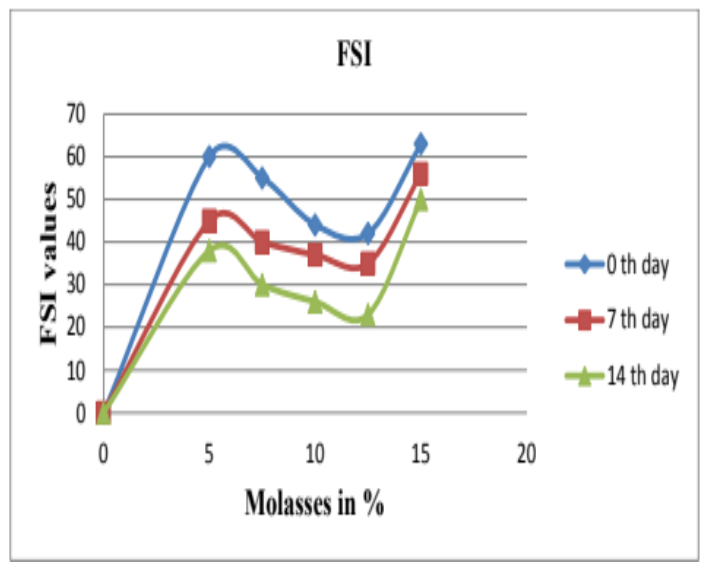

Fig. 1 FSI for treated soil @ 0,7 and 14days curing periods

\section{B. Variation of Atterberg's limits in Unconfined Compressive Strength Test}

It can be observed from table that the Atterberg's limit increase from initial percentage to $12.5 \%$. And there is sudden decrease in Atterberg's limit at reaching 15\%.

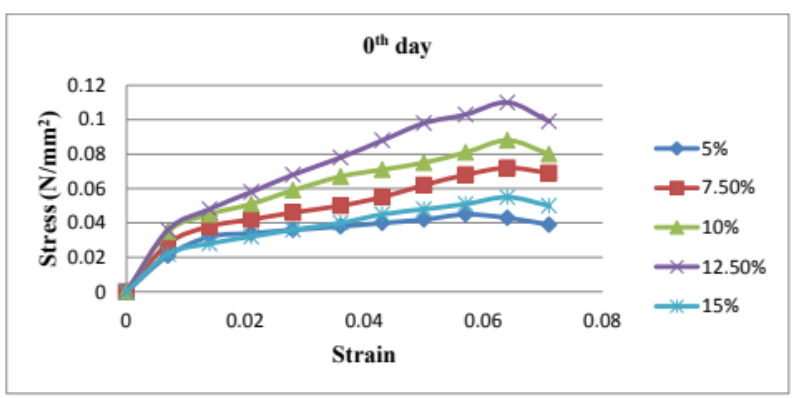

Fig. 2 UCS of treated soil for $0^{\text {th }}$ day

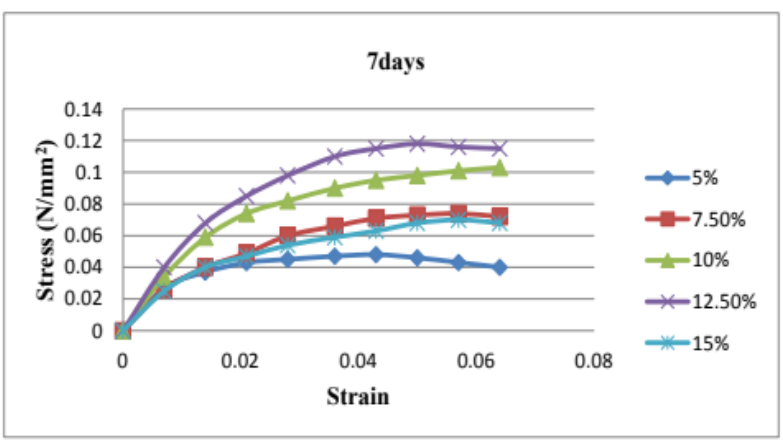

Fig. 3 UCS of treated soil for 7 days curing period

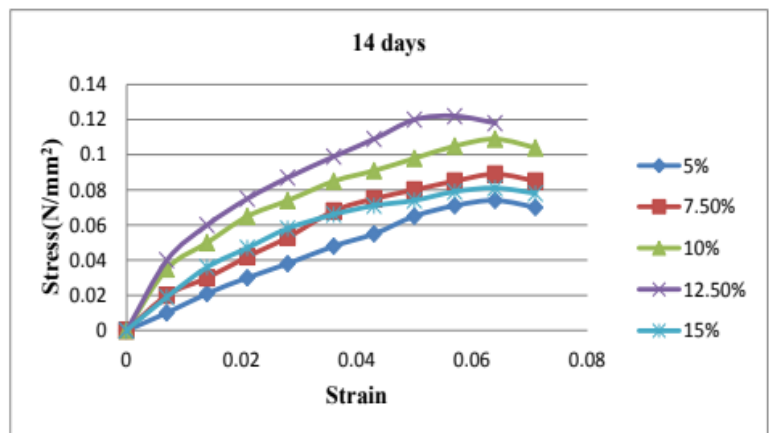

Fig. 4 UCS of treated soil for 14 days curing period

\section{Atterberg's Liquid Limit and Plastic Limit}

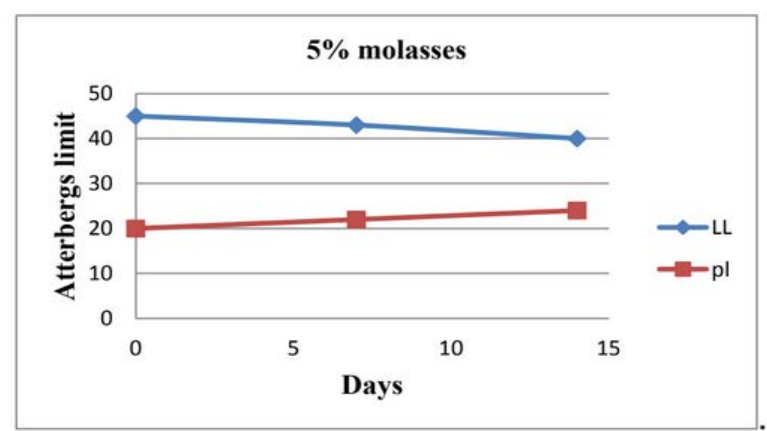

Fig. 5 Liquid limit \& Plastic limit for 5\% of molasses 
From the above figure, Liquid Limit value decreases from $45 \%$ to $40 \%$ with increases in $5 \%$ molasses content deduced that the flow characteristics soil sample and plastic limit of these mixes are increases from $20 \%$ to maximum of $24 \%$.

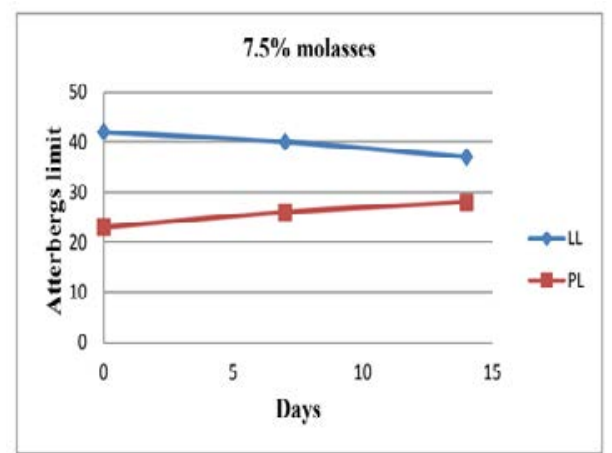

Fig.6 Liquid limit \& Plastic limit for $7.5 \%$ of molasses

From the above figure, Liquid Limit value decreases from $42 \%$ to $37 \%$ with increases in $7.5 \%$ molasses content deduced that the flow characteristics soil sample and plastic limit of these mixes are increases from $23 \%$ to maximum of $28 \%$.

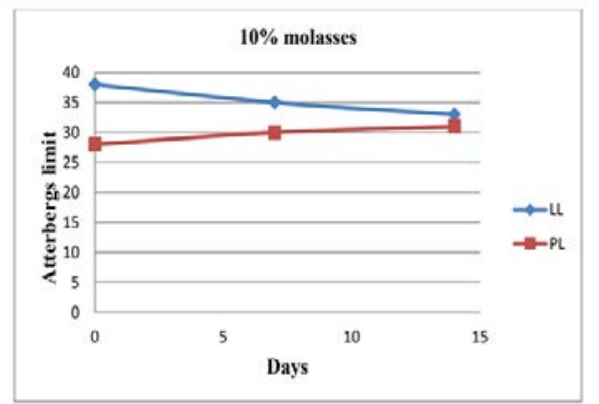

Fig. 7 Liquid limit \& Plastic limit for $10 \%$ of molasses

From the above figure, Liquid Limit value decreases from $38 \%$ to $33 \%$ with increases in $10 \%$ molasses content deduced that the flow characteristics soil sample and plastic limit of these mixes are increases from $28 \%$ to maximum of $31 \%$.

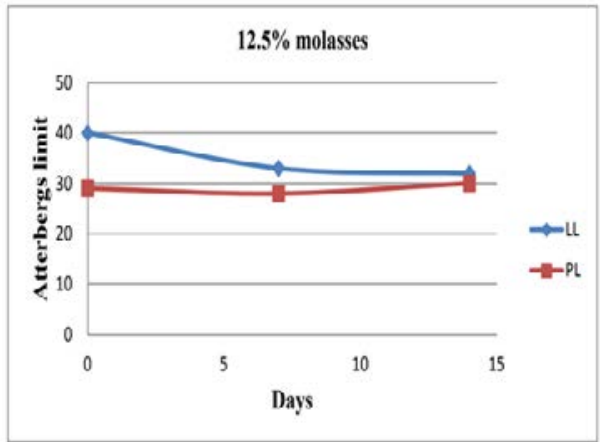

Fig. 8 Liquid limit \& Plastic limit for $12.5 \%$ of molasses

From the above figure, Liquid Limit value decreases from $40 \%$ to $32 \%$ with increases in $12.5 \%$ molasses content deduced that the flow characteristics soil sample and plastic limit of these mixes are increases from $29 \%$ to maximum of $30 \%$.

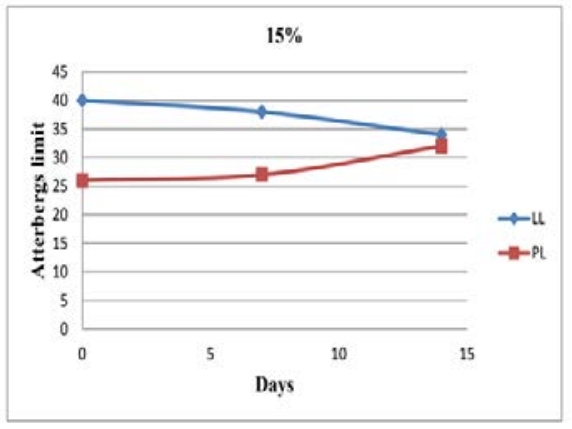

Fig. 9 Liquid limit \& Plastic limit for 15\% of molasses

From the above figure, Liquid Limit value decreases from $40 \%$ to $34 \%$ with increases in $15 \%$ molasses content deduced that the flow characteristics soil sample and plastic limit of these mixes are increases from $26 \%$ to maximum of $32 \%$.

\section{CONCLUSION}

It is observed from the above content that the compressive values are getting increased up to $10 \%$ in free swell index and UCC test. And Atterberg's plastic states were increase slowly and liquid state was vice versa.

\section{REFERENCES}

[1] S. Vinodhkumar and S. Balaji, "Aartificial neural network modelling and economic analysis of black cotton soil subgrade stabilized with flyash and geotextile", International journal of earth sciences and engineering, Vol. 9, No. 01, pp. 81-86, 2016.

[2] Neva Elias, "Strength Development of Soft Soil Stabilized with Waste paper sludge”, International Journal of Advanced Technology in Engineering and Science, Vol. 03, No. 01, January 2015.

[3] M.A'dams joe and A. Maria rajesh, "Soil Stabilization using Industrial Waste and Lime”, International Journal of Scientific Research Engineering \& Technology (IJSRET), Vol. 4, No. 7, July 2015.

[4] Zamri bin chik et al., "Stabilization of Silty Clay Soil using Chloride Compounds", Journal of engineering science and technology, Vol. 2, No. $1,2007$.

[5] S. Vinodhkumar, D. Ramya and P. Kulathaivel, "Index And Chemical Properties Of Black Cotton Soil Blended With Flyash", International Journal of Research in Advanced Technology, Vol. 1, No. 6, 2016.

[6] K. Mahendran, "Utilization of Hypo Sludge for the Stabilization of Red Soils along with Cement and Molasses", Indian Journal of Science and Technology, Vol. 9, No. 2, January 2016.

[7] Malhotra, Monica and Sanjeev Naval, "Stabilization of expansive soils using low cost materials”, International Journal of Engineering and Innovative Technology, Vol. 2, No. 11, 181-184, 2013.

[8] K. Divya Krishnan, "Study on Behaviour of Soil with Phosphogypsum as Stabiliser", Indian Journal of Science and Technology, Vol. 9, No. 23, 2016.

[9] Chakraborty, Arunav, Archita Borah and Debangana Sharmah, "Stabilization of Expansive Soil using Sugarcane Straw Ash (SCSA)”, ADBU Journal of Engineering Technology, Vol. 4, 2016.

[10] D. Ramya and S. Vinodhkumar, "Development of Support Vector Machine Model to Predict Stability of Slopes Based on Bound Theorems", International Journal of Engineering and Technology, Vol. 9, No. 2, Apr-May 2017.

[11] K. Mallikarjuna Rao and G. V. Rama Subbarao, "Optimum fly ash for mechanical stabilization of expansive soils using 22 factorial experimental design”, Natural hazards, Vol. 60, No. 2, pp. 703-713, 2012. 